\title{
SPECTRAL-SPATIAL CLASSIFICATION FOR HYPERSPECTRAL IMAGE BY BILATERAL FILTERING AND MORPHOLOGICAL FEATURES
}

\author{
Wenzhi Liao ${ }^{1}$, Daniel Erick Ochoa Donoso ${ }^{2}$, Frieke Van Coillie ${ }^{3}$, Jie Li ${ }^{1,4}$, Chun Qi ${ }^{4}$, \\ Sidharta Gautama ${ }^{1}$, Wilfried Philips ${ }^{1}$ \\ ${ }^{1}$ Ghent University-TELIN-IPI-iMinds, B-9000 Ghent, Belgium \\ \{Wenzhi.Liao, Sidharta.Gautama, Philips\}@telin.ugent.be \\ ${ }^{2}$ Facultad de Ingeniera en Electricidad y Computacin, Escuela Superior Politcnica del Litoral (ESPOL) \\ Campus Gustavo Galindo, Km. 30. 5 via Perimetral, Apartado 09-01-5863, Guayaquil, Ecuador \\ dochoa@fiec.espol.edu.ec \\ ${ }^{3}$ FORSIT, Department of Forest and Water Management, Gent University, B-9000 Ghent, Belgium \\ frieke.vancoillie@ugent.be \\ ${ }^{4}$ School of Electronics \& Information Engineering, Xi' an Jiaotong University, Xi' an 710049, China \\ \{jiejlixjtu, qichun\}@xjtu.edu.cn
}

\begin{abstract}
Hyperspectral (HS) imagery contains a wealth of spectral and spatial information that can improve target detection and recognition performance. Conventional spectral-spatial classification methods cannot fully exploit both spectral and spatial information of HS image. In this paper, we propose a new method to fuse the spectral and spatial information for HS image classification. Our approach transfers the spatial structures of the whole morphological profile into the original HS image by using bilateral filtering, and obtains an enhanced HS image enriching both spectral and spatial information. Meanwhile, the enhanced HS image has the same spectral and spatial dimensions as the original HS image, which may provide a new input to improve the performances of existing HS image classification methods. Experimental results on real HS images are very encouraging. Compared to the methods using only single feature and stacking all the features together, the proposed fusion method improves the overall classification accuracy more than $10 \%$ and $5 \%$, respectively.
\end{abstract}

Index Terms - Hyperspectral images, data fusion, mathematical morphology, bilateral filtering

\section{INTRODUCTION}

Recent advances in sensors technology have led to an increased availability of hyperspectral data at very high spatial and spectral resolutions. A general framework typically used for the spectral-spatial classification of hyperspectral images

This work was supported by the FWO (Fund for Scientific Research in Flanders) project G037115N: Data fusion for image analysis in remote sensing. either exploits neighbourhood information [2] or deduces the spatial information by mathematical morphological [1, 3](or their variants). However, these approaches have some limitations: (1) the exploitation of neighbourhood information within a sliding window [2] will increase the computational burden, because each pixel is repeatedly treated as a collaborative assistant for its surrounding pixels; (2) morphological profiles (MPs) (or their variants) are built by only considering few components extracted from the original HS cube, hence not fully exploiting the spectral information.

Combining spatial and spectral information can contribute to a more comprehensive interpretation of objects on the ground. For example, spectral signatures cannot differentiate between objects made of the same material (e.g., roofs and roads made with the same asphalt), while they can often be easily distinguished by their geometry. On the other hand, spatial features alone may fail to discriminate between objects that are quite different in nature (e.g., grass field, parking, or a swimming pool), if their shape and size are similar. Many approaches have been developed to fuse the spectral and spatial information for the classification of remote sensing data $[3,4,5,6]$. Some of these approaches employ the so-called composite kernel methods [4] or their generalization [5]. Others define spatial information through MPs, and concatenate spectral and spatial features in a stacked architecture for classification [6]. In our recent work [3], we defined a local fusion graph to couple dimension reduction and data fusion of spectral and spatial features, demonstrating an improvement in classification. However, these approaches treat spectral and spatial features individually, and suffer the problem of spectral and spatial dimension selection.

In this paper, we propose a novel spectral-spatial fusion 
framework for hyperspectral image classification by using bilateral filtering. Specifically, we first extract spatial information by computing the extended morphological profile (EMP) on original hyperspectral image. We then transfer the spatial relationships of EMP to the original hyperspectral image by bilateral filtering [7], and obtain an enhanced hyperspectral image without increasing or reducing spectral and spatial dimensionalities. Last but not least the enhanced HS image is used as the input of SVM classifier to get the final classification map. Experimental results demonstrate the proposed spectral-spatial fusion method can transfer complementary spatial information into the original hyperspectral image, and enable better performances on classification. The remainder of the paper is organized as follows. The proposed spectral-spatial fusion method is detailed in Section 2. Section 3 presents the experimental results. Finally, conclusions are drawn in Section 4.

\section{METHODOLOGY}

\subsection{Morphological features}

Typical morphological features used for characterizing the spatial information of very high resolution remote sensing images are generated by applying morphological openings and closings by reconstruction [1] on a grayscale image, using a structural element (SE) of predefined size and shape. An opening acts on objects that are bright compared with their surrounding, while closings act on dark objects. For example, an opening deletes (i.e. the pixels in the object take on the value of their surrounding) bright objects that are smaller than the SE. By increasing the size of the SE and repeating the previous operation, a complete morphological profile is built [1], carrying information about the size and the shape of objects in the image due to its multiscale nature.

When applying MP to hyperspectral data, principal component analysis (PCA) is widely used as a pre-processing step to reduce the dimensionality of the high-dimensional original data, as well as to reduce redundancy among the bands [1]. Then one constructs a morphological profile on each principal component (PC) independently. An extended MP (EMP) is formed by stacking all the computed morphological profiles. Specifically in our experiments, the first 3 PCs (representing more than $99 \%$ of the cumulative variance) of original HS image are used to computed EMP, with 4 openings and closings (ranging from 2 to 8 with step size increment of 2). The usefulness of using morphological features to exploit the spatial information of high-resolution remote sensing images has been discussed in numerous studies $[3,1,6]$.

\subsection{Proposed spectral-spatial fusion method}

Extensive research has been conducted to transfer the spatial information into hyperspectral imagery, e.g., hyperspectral image pansharpening $[9,10]$. The objective of these ap- proaches is to obtain an enhanced hyperspectral image, with the optimized preservations on both spectral and spatial information. However, they mainly focus on boundary spatial information transformation from an additional image source. This paper will transfer the spatial relationships of a complementary features to the original hyperspectral image. The complementary features are generated from the original image, which includes not only boundary information, but also object size and shape information of the image. We take bilateral filter [7] as an example, as its efficient and strong abilities to transfer the spatial relationships of the guidance image to the filtering output. Its application to HS data can be found in [8], where the bilateral filter [7] was applied to transfer the structures of the principal components of the HS image to the initial classification maps.

Our proposed framework for spectral and spatial fusion exploits the transformation of the morphological information to a HS image by using bilateral filter [7] in PCA domain. Instead of simply stacking or graph fusion spectral and spatial features [3] which may not be easy to be interpreted, our proposed fusion method obtains an enhanced HS image enriching both spectral and spatial information while keeping the spectral and spatial dimensionalities the same to the original HS image. This way, our proposed method may provide an enhanced input for existing HS image processing techniques. By joint bilateral filtering the spectral and spatial fusion process, our method can not only preserve the spectral information from the original HS image, but also transfer the spatial structures (e.g., size, shape) of the EMP to the enhanced HS image. To reduce the noise effect, our method first uses PCA to decorrelate the HS images and separate the information content from the noise. The first $k$ PCA channels contain most of the total energy of a HS image (i.e., most information of the HS image), and the remaining $B-k$ PCA channels (where $B$ is the number of spectral bands of HSI and $B \gg k$ ) mainly contain noise. If joint bilateral filtering is performed on these noisy and high-dimensional $B-k$ PCs, then it will amplify the noise of the data cube, which is undesirable. Therefore, we only transfer the spatial structures of EMP to the first $k$ PCA channels by using bilateral filter. Let $P C_{i} \in \Re^{k}$ and $P C_{j} \in \Re^{k}$ denote the $i$ th pixel and its neighboring pixels of the first $k$ PC (of original HS image), the filtering output $P C_{i}^{\prime} \in \Re^{k}$ can be represented as a linear transform of guided profile EMP in a local window of size $\left(2 \delta_{s}+1\right) \times\left(2 \delta_{s}+1\right)$ as follows:

$$
P C_{i}^{\prime}=\frac{1}{K_{i}} \sum_{j \in \omega_{i}} G_{\delta_{s}}(\|i-j\|) G_{\delta_{r}}\left(\left|E M P_{i}-E M P_{j}\right|\right) P C_{j}
$$

$K$ is a normalizing term of the joint bilateral filter:

$$
K_{i}=\sum_{j \in \omega_{i}} G_{\delta_{s}}(|| i-j \|) G_{\delta_{r}}\left(\left|E M P_{i}-E M P_{j}\right|\right)
$$

where $\delta_{s}$ controls the size of the local window used to filter 
a pixel, and $\delta_{r}$ defines how much the weight of a pixel decreases because of the feature difference between the guided profile pixels (i.e., $E M P_{i}$ and $\left.E M P_{j}\right) . G_{\delta_{s}}(\| i-$ $j \|)=\exp \left(-\|i-j\| / \delta_{s}^{2}\right)$ and $G_{\delta_{r}}\left(\left|E M P_{i}-E M P_{j}\right|\right)=$ $\exp \left(-\left|E M P_{i}-E M P_{j}\right|^{2} / \delta_{r}^{2}\right)$ are spatial and range kernels defined using two Gaussian decreasing functions.

The kernels are the combination of weights based on both the spectral similarity and spatial similarity. From equations (1) and (2), we can easily find that if the neighboring pixels of pixel $i$ in the EMP have similar characteristics (i.e., $E M P_{i} \approx E M P_{j}$ ), the weight of pixel $j$ (represented by neighboring pixel $j$ ) will be quite large, especially when it is very geometrically close to $i$ (i.e., $\|i-j\|$ is very small). On the contrary, if the neighboring pixels have quite different characteristics in the guidance profile, the situation will be the opposite. This means that the filter will only smooth spectra with similar characteristics on both spectral and spatial domains and keep the rest untouched. This way our proposed method ensures the preservation of the original spectral information while transferring the spatial information of EMP. For more details about bilateral filter, we refer the readers to [7]. We remove the noise in the remaining PCA channels using a soft-thresholding scheme (and without joint bilateral filter). We obtain the enhanced HS image by inverting PCA transformation.

\section{EXPERIMENTAL RESULTS}

Experiments are done on a hyperspectral data of Pavia University, which was collected by the Reflective Optics System Imaging Spectrometer (ROSIS-03). This data set has 115 bands, with a spectral range of 0.43-0.86 um and very fine spatial resolution of $1.3 \mathrm{~m}$ per pixel. After removing 12 water absorption and noisy bands, 103 bands were used in the experiments. This data set includes nine land cover/use classes, see Table 1. Note that the color in the cell denotes different classes in the classification maps (Fig. 1)

The SVM classifier with radial basis function (RBF) kernels [9] is applied in our experiments. We apply a gridsearch on the two parameters $C$ and $\gamma$ of SVM using 5fold cross-validation to find the best $C$ within the given set $\left\{10^{-1}, 10^{0}, 10^{1}, 10^{2}, 10^{3}\right\}$ and the best $\gamma$ within the given set $\left\{10^{-3}, 10^{-2}, 10^{-1}, 10^{0}, 10^{1}\right\}$. We compare our proposed spectral-spatial fusion method with the schemes of (1) Only using raw hyperspectral data (Raw); (2) Only using extended morphological profile (EMP); (3) Stacking raw hyperspectral data and EMP together (Sta), similar as [6]. The classification results are quantitatively evaluated by measuring the Overall Accuracy (OA), the Average Accuracy (AA) and the Kappa coefficient $(\kappa)$ on the test data. Table 1 shows the accuracies obtained from the experiments, Fig. 1 shows the best result of each method.

It is obvious that using raw hyperspectral is not enough for reliable classification, with relative lower accuracy and
Table 1: Comparison on classification accuracy.

\begin{tabular}{|l|c|c|c|c|}
\hline & Raw & EMP & Sta & Proposed \\
\hline No. of Features & 103 & 27 & 130 & 103 \\
\hline OA (\%) & 79.75 & 80.26 & 85.69 & $\mathbf{9 3 . 3 1}$ \\
AA (\%) & 88.26 & 88.74 & 92.37 & $\mathbf{9 3 . 9 9}$ \\
$\kappa$ & 0.747 & 0.749 & 0.819 & $\mathbf{0 . 9 1 3}$ \\
\hline Asphalt & 84.17 & 93.53 & $\mathbf{9 3 . 8 2}$ & 91.52 \\
Meadows & 67.42 & 69.30 & 74.16 & $\mathbf{9 2 . 3 2}$ \\
Gravel & 73.70 & 73.08 & 72.32 & $\mathbf{7 8 . 7 0}$ \\
Trees & 94.78 & $\mathbf{9 7 . 7 5}$ & 96.93 & 94.13 \\
Metal Sheets & 99.63 & $\mathbf{9 9 . 9 3}$ & 99.85 & 99.55 \\
Soil & 92.30 & 68.48 & 96.78 & $\mathbf{9 8 . 2 7}$ \\
Bitumen & 91.20 & 97.67 & $\mathbf{9 8 . 5 7}$ & 94.21 \\
Bricks & 91.47 & 98.94 & $\mathbf{9 9 . 1 0}$ & 98.34 \\
Shadows & 99.68 & $\mathbf{1 0 0 . 0}$ & 99.79 & 98.84 \\
\hline
\end{tabular}

noisy map. By using only the spatial information through EMP, we produce smoother classification map. However, the remote sensing data from urban area was a mix between manmade structures and natural materials, either spectral or spatial feature alone is not sufficient to discriminate different classes. For example, single hyperspectral image misclassified many objects made with same material, e.g., 'Asphalt'; while EMP alone cannot well separated objects with similar size and shape, like 'Meadows' and 'Soil', leading to poor performances. By simply stacking different feature sources together, we won't always produce better results than using single feature source [6], but increase the dimensionality of the input for the SVM classifier (which will consume more computational time).

Our proposed method enhanced hyperspectral image by exploiting spatial information from EMP. Meanwhile, we also preserve the spectral information of original hyperspectral image, and enable much better classification performances. Compared with using single feature source or simply stacking both of features together, our proposed method has more than $10 \%$ and $5 \%$ improvements in accuracies, respectively.

\section{CONCLUSION}

This paper presents a new spectral-spatial fusion method for hyperspectral image classification. Different from existing methods, our proposed method transfers the spatial structures of the whole morphological profile to original hyperspectral image, without increasing/reducing the spectral and spatial dimensions. By taking into account both spectral and spatial information, our proposed method preserves spectral information while transferring the spatial relationships from complementary features into the resulting enhanced hyperspectral data, and improves classification performances. Experimental results on the classification of a hyperspectral image show 


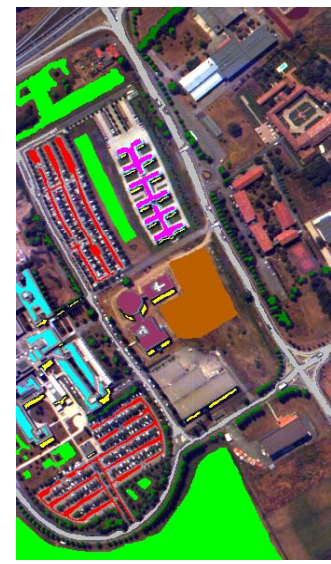

(a) Ground truth

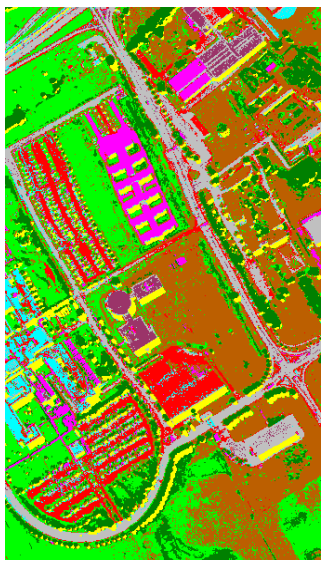

(b) Raw

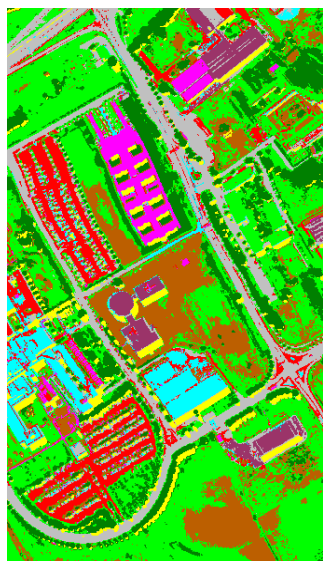

(c) EMP

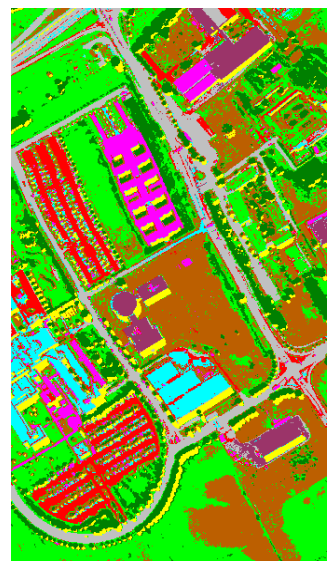

(d) Stacking

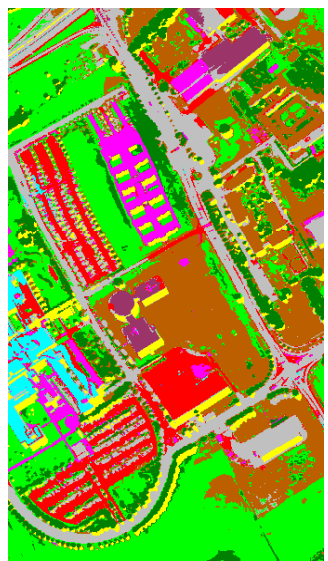

(e) Proposed

Fig. 1: classification maps obtained by each method.

the efficiency of the proposed method, with significant classification accuracy improvements over using single feature and simply stacking different features together. Our approach may provide an enhanced input to improve the performances of the existing hyperspectral image classification methods.

\section{Acknowledgment}

The authors would like to thank Prof. P. Gamba from the University of Pavia, Italy, for kindly providing the hyperspectral data of University area.

\section{REFERENCES}

[1] J. A. Benediktsson, J. Palmason, and J. R. Sveinsson, "Classification of hyperspectral data from urban areas based on extended morphological profiles", IEEE Trans. Geosci. Remote Sens., vol. 43, no. 3, pp. 480-491, Mar. 2005.

[2] Y. Chen, Z. Lin, X. Zhao, G. Wang, "Deep Learningbased Classification of Hyperspectral Data", IEEE J. Select. Top. Appl. Earth Observ. Remote Sens., vol. 7, no. 6, pp. 2094-2107, 2014.

[3] W. Liao, M. Dalla Mura, J. Chanussot, A. Pizurica, "Fusion of Spectral and Spatial Information for Classification of Hyperspectral Remote Sensed Imagery by Local Graph", IEEE J. Select. Top. Appl. Earth Observ. Remote Sens., vol. 9, no. 2, pp. 583-594, Feb. 2016.

[4] G. Camps-Valls, L. Gomez-Chova, J. Munoz-Mari, J. Vila-Frances, and J. Calpe-Maravilla, "Composite kernels for hyperspectral image classification," IEEE Geosci. Remote Sens. Lett., vol. 3, no. 1, pp. 93-97, Jan. 2006.

[5] J. Li, P. R. Marpu, A. Plaza, J. M. Bioucas-Dias and J. A. Benediktsson, "Generalized Composite Kernel Framework for Hyperspectral Image Classification", IEEE
Trans. Geosci. Remote Sens., vol. 51, no. 9, pp. 48164829, Sep. 2013.

[6] M. Fauvel, J. A. Benediktsson, J. Chanussot and J. R. Sveinsson, "Spectral and Spatial Classification of Hyperspectral Data Using SVMs and Morphological Profile", IEEE Trans. Geosci. Remote Sens., vol. 46, no. 11, pp. 3804-3814, Nov. 2008.

[7] S. Paris and F. Durand, "A fast approximation of the bilateral filter using a signal processing approach", Int. J. Comput. Vis., vol. 81, no. 1, pp. 24-52, Jan. 2009.

[8] X. Kang, S. Li, J. A. Benediktsson, "Spectral-spatial hyperspectral image classification with edge-preserving filtering",IEEE Trans. Geosci. Remote Sens., vol. 52, no. 5, pp. 2666-2677, May, 2014.

[9] W. Liao, X. Huang, F. Coillie, S. Gautama, A. Pizurica, W. Philips, H. Liu, T. Zhu, M. Shimoni, G. Moser, and D. Tuia, "Processing of Multiresolution Thermal Hyperspectral and Digital Color Data: Outcome of the 2014 IEEE GRSS Data Fusion Contest", IEEE J. Select. Top. Appl. Earth Observ. Remote Sens., vol. 8, no. 6, pp. 29842996, June 2015.

[10] L. Loncan, L. B. Almeida, J. Bioucas Dias, X. Briottet, J. Chanussot, N. Dobigeon, S. Fabre, W. Liao, G. A. Licciardi, M. Simoes, J. Y. Tourneret, M. A. Veganzones, G. Vivone, Q. Wei, and N. Yokoya, "Hyperspectral pansharpening: a review", IEEE Geoscience and Remote Sensing Magazine, vol. 3, no. 3, pp. 27-46, Sep. 2015.

[11] W. Liao, M. Dalla Mura, J. Chanussot, R. Bellens, W. Philips, "Morphological Attribute Profiles With Partial Reconstruction", IEEE Trans. Geosci. Remote Sens., vol. 54, no. 3, pp. 1738-1756, Feb. 2016. 